Fotoficção e Fotojornalismo

A 



\title{
Pour un Partage DU VISIBLE Mes Algéries en France, de Leīla Sebbar
}

\author{
SEWING TOGETHER SHARED ICONS: LEÏLA SEBBAR'S \\ MES ALGÉRIES EN FRANCE
}

\author{
Jean-Pierre Montier* \\ Université Rennes
}

\begin{abstract}
RÉ S U MÉ
Dans Mes Algéries en France, Leïla Sebbar use de la forme de l'iconotexte pour son hybridité, miroir de sa propre «bigarrure» généalogique. Tel un gant que l'on retournerait, elle se sert de ce genre «bâtard» pour affirmer la nécessité conjointe de suturer les blessures que l'histoire a ouvertes entre les familles des deux rives de la Méditerranée, et de penser le récit d'une vie singulière comme lié, «cousu», à celui de toutes les autres vies. Dès lors l'écriture de l'Histoire et l'autobiographie littéraire convergent-elles dans ce livre singulier pour inventer une nouvelle formule de l'autobiographie, que je nomme ici «alterégotiste», qui est peut-être particulière à la figure de la femme écrivain, mais qui est peut-être également liée à un usage singulier de la photographie, dont le potentiel de conservation du passé ne concerne pas exclusivement la personne ou la famille, puisqu'on voit Leïla Sebbar l'utiliser de manière à circonscrire un véritable «lieu de mémoire» collectif, au sens où l'historien Pierre Nora emploie ce terme.
\end{abstract}

\section{MOTS-CLÉS}

Autobiographie, iconotexte, post-colonial, Leïla Sebbar, photolittérature

Mes Algéries en France, de Leïla Sebbar, paru en 2004 chez Bleu Autour, est une sorte d'objet littéraire non identifié: des libraires ont eu quelque peine à en comprendre les caractéristiques, puisque je puis témoigner que le mien l'avait classé dans le rayon consacré au tourisme, bien que son auteur soit désormais bien connue comme écrivain.

En effet, à considérer le livre dans sa matérialité, rien n'y indique expressément qu'il s'agisse d'une forme d'autobiographie. Le titre de la collection dans laquelle il s'inscrit, «D'un regard l'autre», renvoie à l'abondance du corpus iconographique et à la thématique de l'altérité culturelle que l'on retrouve notamment dans un autre livre, Femmes d'Afrique du Nord, où Jean-Michel Belorgey et Leïla Sebbar ont collecté un

\footnotetext{
* jean-pierre.montier@uhb.fr
} 
corpus de cartes postales anciennes. En outre, le sous-titre, «Carnet de voyages», est en trompe-l'œil: l'ouvrage ne relève ni de la veine viatique ni des souvenirs d'excursion. Si la préface rédigée par l'historienne Michelle Perrot évoque bien les «routes algériennes de Leïla Sebbar», c'est pour mieux souligner qu'il sera question d'un itinéraire au sens métaphorique. Cette «route» est évidemment une image à la fois de la «vie» et de l'écriture, largement employée dans la littérature romanesque, mais, malgré le pronom personnel de première personne (Mes Algéries), ce «Carnet de voyages» n'équivaut pas à «récit de vie», sinon sur le mode de la devinette: sur la couverture, entourant le titre, figurent, à la façon d'un puzzle, quatorze photographies et dessins de personnages célèbres ou inconnus évoquant l'Algérie coloniale, dont le plus immédiatement reconnaissable est Zinnedine Zidane. On n'a pas, à première vue, de portrait de l'auteur comme telle. Cependant, à bien y regarder, le lecteur découvrira en parcourant l'ouvrage (page 221) que la photo qui, en haut, à gauche de la couverture, montre une petite fille d'une dizaine d'années, aux cheveux retenus par deux rubans blancs, est celle de Leïla Sebbar enfant. La couverture dit déjà tout: l'auteur y est, mais en enfant, et à discerner parmi d'autres visages... Un jeu de devinette, qui est fait pour mettre sur des pistes.

\section{DE QUOI S'AGIT-IL?}

Mon hypothèse de travail consistera à considérer cette couverture de Mes Algéries en France (FIG. 1) comme la scène à partir de laquelle Leïla Sebbar brouille les catégories génériques distinguant le récit de l'historien et celui du témoin, l'album de famille et les archives nationales, les personnages de fiction et ceux de l'histoire, les sphères du public et du privé, l'évocation des souvenirs les plus personnels et celle de l'actualité la plus fraîche, de telle sorte que nous aboutissons à une formulation neuve et originale de l'autobiographie, entendue non plus comme l'exposition d'un ego selon le fil du temps d'une vie individuelle, mais comme le déploiement d'un iconotexte, certes personnel et subjectif, mais ouvert à l'investissement d'une pluralité d'autres sujets, avec, en perspective, l'esquisse d'une histoire plurielle. Autrement dit, la couverture est un leurre qui fonctionne parfaitement: ce n'est ni un guide touristique, ni un album nostalgique, ni la nième variation sur la question - vaine, sinon «meurtrière» pour reprendre Maalouf - de l'identité coloniale: il s'agit d'un authentique ouvrage icono-littéraire grâce auquel se marque un déplacement manifeste des enjeux relatifs à la notion même d'autobiographie, pensée comme un prisme où viennent se réfracter mémoire de soi et histoire collective.

Il faut donner ici quelques éléments concernant cet auteur essentiel. Leïla Sebbar est née en novembre 1941 à Aflou, d'un père algérien et d'une mère française, tous deux instituteurs. Elle fait une hypokhâgne au lycée Bugeaud en 1959, quitte l'Algérie à dix-huit ans pour Aix-en-Provence, puis Paris, où elle fait des études supérieures en Lettres. Elle enseigne dans un lycée et collabore à diverses revues, dont Les Temps Modernes et La Quinzaine Littéraire. Ses recherches porteront sur les représentations du bon nègre et sur les violences faites aux filles. Leïla Sebbar s'emploie dans toutes ses œuvres à recoudre la déchirure entre l'Algérie et la France. La métaphore de la couture - travail féminin dans la culture méditerranéenne, au moins depuis Pénélope! - est humoristiquement présente dans Mes Algéries en France, avec une publicité pour la machine 
à coudre de marque «Singer», apposée sur un mur de Paussac, dans le Périgord. ${ }^{1}$ Mère périgourdine, père algérien: les femmes recousent ce que les hommes mettent en pièces... Le roman pour lequel elle a obtenu en 1993 le Prix Kateb Yacine, Le silence des rives, pose une parole qui formule à la fois la déchirure douloureuse et la profondeur des liens multiples. Avec La Seine était rouge, Paris, octobre 1961, publié chez Thierry Magnier en 1999, elle aborde de front l'un des multiples épisodes tragiques d'une mémoire qui a toutes les peines à se formuler dans un langage qui serait commun et ne rouvrirait pas les blessures. Ses ouvrages, publiés aussi par des éditions nord-africaines, comportent souvent une iconographie substantielle. C'est ainsi pour Les Algériens au café, avec des dessins de Sébastien Pignon, Al Manar, 2003; Isabelle l'Algérien, Al Manar, 2005; Algérie, textes et dessins inédits pour l'Algérie, Casablanca, 1995. Par féminisme, mais surtout parce que Leïla Sebbar est convaincue que, dans la constitution d'une mémoire apaisée, les femmes ont un rôle essentiel à jouer, c'est aussi à la situation des femmes algériennes ou africaines des banlieues françaises qu'elle s'intéresse, avec notamment un recueil de nouvelles accompagnées de photographies, Val nord, fragments de banlieue, publié par Gilles Larvor en 1998. Le choix de mêler fréquemment texte et image correspond à une nécessité forte, renvoyant à la définition que Leïla Sebbar donne d'elle-même. Dans une nouvelle intitulée Les jeunes filles de la colonie, elle se dit bigarrée: «D'abord ce n'est pas la guerre. Et je ne sais pas que je suis bigarrée.». ${ }^{2}$ La bigarrure, qui caractérise un iconotexte, est l'expérience de soi dans laquelle, comme sujet, elle prend son origine, dont elle tire son originalité d'écrivain et le genre littéraire qu'elle adopte. La notion d'iconotexte prend alors toute sa force, puisqu'il ne s'agit pas d'illustrer des mots avec des images mais bien d'inventer une forme, aussi impure ou bâtarde, que l'on voudra, mais en laquelle vient s'imposer l'ardente obligation d'en finir avec les non-dits et de reformuler la mémoire en un langage, mots et images croisés, commun aux hommes et femmes des «deux rives».

\section{UNE FIGURE D'AUTEUR À REBOURS DES REVENDICATIONS IDENTITAIRES}

Superficiellement, Leïla Sebbar pourrait incarner un cas d'interculturalité, un métissage lisse et sans aspérités. Mais elle récuse aussi bien les idéologies irénistes que les postures nourrissant le ressentiment. Pour elle, le préfixe inter n'a pas fonctionné entre les cultures dont elle est issue, un adversatif «mais» s'est constamment glissé entre elles. Sa mère était institutrice, mais femme et française à la peau blanche. Leïla est la seule de la fratrie que l'on n'appelait que par son prénom arabe, contrairement à son frère Alain et à ses sœurs, Danièle et Lysel; mais la langue de son père lui est demeurée étrangère. Son père - produit d'une méritocratie théorique, mais tempérée par le statut colonial - était chargé d'enseigner le français, mais aux jeunes «indigènes»; il se vivait comme républicain et laïque, mais algérien et musulman. Un père minoré du fait de son origine, mais valorisé comme détenteur de savoir (il est passé par l'École normale d'instituteurs de Bouzaréah à Alger, comme son ami Mouloud Feraoun), qui

\footnotetext{
${ }^{1}$ SEBBAR. Mes Algéries en France, p. 65.

${ }^{2}$ SEBBAR. Une enfance outremer, p. 187.
} 
sera perçu comme un suppôt des Français par les maquisards du FLN mais recherché aussi par l'armée française. On pourrait ainsi remonter en cascade l'échelle des contradictions jusqu'au système colonial français entier, qui prétendait reposer sur des principes universalistes que leur mise en œuvre contredisait systématiquement.

Aussi bien, la figure du «père dont elle ne parle pas la langue» (Je ne parle pas la langue de mon père est paru chez Julliard en 2003) ne me semble relever que superficiellement de catégories psychologiques. Leïla Sebbar n'entretient nul grief envers celui dont elle dresse un portrait en chevalier courtois qui séduit sa future mère lors d'un bal, au cours d'un stage de jeunes normaliens organisé dans le Périgord: «Lorsqu'elle revient à la table de ses amies et qu'il s'assoit près d'elle, elle le regarde. Ses cheveux sont noirs et frisés. Il a les yeux bleus. Outremer. Il l'enlève. [...] Je suis née d'un enlèvement d'amour.». ${ }^{3}$ Sa mère n'était pas issue d'une famille de colons, son mariage n'était pas une mésalliance réprouvée entre Français de souche et indigène, il déjouait la relation entre dominant et dominé, instaurait une réciprocité d'alliance matrimoniale, inouïe sans doute à l'époque, mais anticipant peut-être (Camus et d'autres en avaient rêvé) une vraie réciprocité entre peuples et terres, de part et d'autre de la Méditerranée. Dans sa biographie, Leïla Sebbar puise son «utopie», du moins ses convictions. Demeure pourtant le côté parfaitement intempestif de cette alliance dans laquelle le choix que font deux êtres l'un de l'autre ne saurait être entièrement approuvé par le corps social auquel ils appartiennent, ni surtout validé par le cours pris par l'histoire. Contrairement à Figaro, Leïla Sebbar ne se prétend pas «supérieure aux événements». Elle n'apprendra pas cette langue que son père n'a pas voulu lui transmettre, c'eût été aller contre sa volonté, refaire le cours de choses artificiellement. Larabe, il ne lui en reste que le souvenir de sa musicalité, mais aussi celui des injures que proféraient parfois les garçons qui la croisaient lorsqu'elle était en jupe. Pour être elle-même, Leïla Sebbar devra maintenir la déchirure entre les langues maternelle et paternelle, y puiser le dessein d'en constituer une tierce langue, qu'on appelle communément «littérature». Si pathologie il y a, et si l'ignorance de la langue paternelle en est un symptôme, ce dernier est parfaitement assumé, et même retourné en faisant de cette faille une force.

Et si l'entreprise autobiographique est nécessairement personnelle, Leïla Sebbar prend le soin de ne pas en faire une question strictement individuelle, de conserver la dimension d'un «cas d'école» que son roman familial a pour un historien ou un sociologue. Elle s'est immunisée contre l'illusion qui eût consisté à considérer sa propre «destinée» comme unique, ni lié à sa seule personnalité.

Mes Algéries en France présente en ses dernières pages une table des matières, comme un livre universitaire, commençant par l'enfance, «Portrait de famille; les écoles», puis proposant des synthèses historiques. «Algériennes» est une suite d'hommages aux femmes; «Arts et lettres» évoque quelques grandes figures de l'histoire et de la littérature telles qu'Abd el-Kader, Isabelle Eberhardt, Mouloud Feraoun, Mohamed Dib, Jean Pélégri, Kateb Yacine, mais aussi Zidane, pour une saynète racontée par la mère de l'artiste en ballon rond, écrite par Leïla Sebbar à l'occasion d'une exposition en 2003 à Marseille. Les héros de la quatrième partie, «Une passion algérienne», sont Germaine Tillion, Marthe Stora,

\footnotetext{
${ }^{3}$ SEBBAR. Mes Algéries en France, p. 25.
} 
Aimée Chouraqui, Michèle Perrot, Josette Audin, Pierre Vidal-Naquet. Ensuite, viennent trois chapitres à valeur de mémorial: «Les hommes assis», consacré aux Chibanis - une terme affectueux qu'elle fait subrepticement entrer dans la langue française -, puis «Le champ des morts», qui évoque les soldats des colonies, morts pour la France; enfin «Parcs et jardins; bestiaire», à la tonalité plus intimiste à nouveau. Suivent deux index, des noms de personnes et de lieux: écrire l'histoire et faire de la littérature deviennent ici un seul et même acte, collectif. Raison pour laquelle l'ouvrage est fait de textes de Leïla Sebbar, et d'autres textes qui ne sont pas d'elle: les propos de Pierre Vidal-Naquet et Michèle Perrot, repris d'interview données à la revue Algérie/Littérature/Action; ceux d'Aimée Chouraqui ont été publiés dans Histoires d'elles. Autant de vies parallèles à celle de Leïla Sebbar, intégrées à la sienne, indissolublement. Les textes pris en charge par l'auteur se présentant tantôt comme des souvenirs, tantôt comme des fictions, avec des personnages derrière lesquels se devinent de vraies vies, ayant valeur de symboles, telle Shérazade (p. 73-75), qui est à la fois une «beurette» et une projection de l'Odalisque en rouge, de Giraud, reproduite en pleine page, une incarnation de toutes les femmes rêvées jadis par les Orientalistes. Le personnage est associé à la figure double de Hatidjé/Aziyadé, avec un portrait de Pierre Loti (p. 150-51) placé en regard d'une photographie intitulée «Chez moi, Paris XIIIe, années 90» sur laquelle, autour d'une photo d'enfant, l'on voit divers objets, dont un carton de publicité pour un restaurant dénommé «Sharazad».

Mes Algéries en France ne s'inscrit pas dans la logique traditionnelle, par laquelle le récit de sa propre vie est défini d'une part grâce à l'appartenance au genre narratif, de l'autre en fonction de la catégorie du «bios», d'une vie bornée par la naissance et la mort, scandée par la chronologie d'une maturation allant de l'enfance à l'âge mûr, tendue par la poussée des jours en direction du terme à échoir. L'un des prérequis de l'entreprise autobiographique classique est la nécessité d'un point fixe, permettant une vue panoramique sur sa propre existence, à partir duquel tout vient à s'ordonner. On en trouvera un paradigme dans les dernières lignes des Mémoires d'Outre-tombe de Chateaubriand:

En traçant ces derniers mots, ce 16 novembre 1841, ma fenêtre qui donne à l'ouest sur le jardin des Missions étrangères est ouverte; il est six heures du matin; j'aperçois la lune pâle et élargie; elle s'abaisse sur la flèche des Invalides à peine révélée par les premiers rayons dorés de l'Orient. Je vois se lever les reflets d'une aurore dont je ne verrai pas se lever le soleil.

Or, il n'y a rien d'équivalent, chez Leïla Sebbar, à ce puissant ancrage dans une langue qui fasse d'elle-même autorité, à des lieux officiels (les Invalides) aptes à fédérer un point de vue unique sur les êtres, les événements, ni de «moi» qui soit un point focal privilégié, ni évidemment de sens de l'Histoire qui soit garanti par un Dieu. Au contraire, le lieu en lequel son écriture prend son origine est son appartement parisien, dont de multiples photographies montrent au lecteur les livres de sa bibliothèque, les menus objets collectionnés, les fétiches qu'il contient: un emballage d'oranges «Leïla», les cigognes qui symbolisent l'Alsace mais hibernent en Algérie, etc. Ces images créent une diffraction vers autrui plutôt qu'une confluence vers soi. La dimension personnelle est bien présente, mais de manière subreptice, voilée, puisque le lecteur est amené à saisir l'allusion à la «maison» de la femme auteur après être passé par les références à l'intimité de toutes les femmes musulmanes. Intimités réelles ou fantasmées par les Orientalistes. Intimités contemporaines, vécues dans des banlieues par une autre génération, celle des «beurettes». 


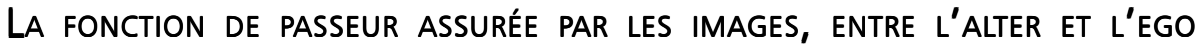

Le dispositif inventé par Leïla Sebbar exclut tout narcissisme, tout lyrisme grandiloquent. Le livre parle de ce qui fâche, sans arrogance ni aigreur. Il fait dialoguer l'individuel et le collectif, sans jugement de valeur, ce qui ne veut pas dire sans fermeté du discours. Tout en récusant le «moi-je», Leïla Sebbar convoque une forme d'intimité renvoyant moins à sa personne qu'au lieu utopique de son écriture: cette langue commune qui est à inventer pour parler ensemble de ce qui sépare et réunit, qui ne saurait être ni le français qui a opprimé, ni l'arabe qu'elle n'a pas appris, langue qu'au demeurant tous les Algériens ne parlent pas, et qui du reste fut jadis aussi une langue de colonisateur.

Mes Algéries en France proposerait donc soit le substitut du manuel d'histoire commune que nul n'a réussi à écrire, soit une autobiographie que j'appellerai «non égocentrique». Pour dépasser ce paradoxe - par définition toute autobiographie est centrée sur un ego, un moi réfléchi par lui-même -, probablement suffit-il de changer la définition du «moi», de sorte qu'il ne serait plus conçu comme une monade, condensant un univers monosémique, mais comme la simple pièce d'un plus vaste puzzle. Ce qui importerait alors ne serait plus l'équation «J'ai pensé ceci, fait cela, donc j'ai été tel ou telle», selon la forme «cartésienne» de l'autobiographie, mais ce serait la manière dont telle pièce - toutes étant différentes quoique d'allure quasi semblable aux autres - s'emboîte dans un ensemble qui primerait. Une définition «non égocentrique» de l'autobiographie impliquerait de concevoir un moi qui n'est que la partie d'un tout dont le dessin (ou dessein) à première vue n'apparaît pas. Chaque élément occupe une place singulière, mais cette singularité doit être patiemment discernée après tout un travail de différentiation, un travail de mémoire. La limite de la métaphore que j'ai employée est dans le fait que, contrairement au puzzle qui contraint à parvenir à une forme unique, ici les pièces sont à emboîtements multiples, dessinent des images superposables; l'important est de chercher de qui se combine, ce qui recoud. De façon positive, je qualifierai ce régime d'«alterégotiste», ou de «polysubjectif». Ainsi, tout en posant son unité et se en démarquant de ses semblables, l'ego impose la nécessité d'une combinaison avec eux, pour dessiner les contours d'une vie qui ne se conçoit pas sans être partie prenante d'un très large faisceau de relations.

Dans L'Arabe comme un chant secret, Leïla Sebbar écrit: «Je ne suis pas une individue, je suis toutes les femmes.» ${ }^{4} \mathrm{Et}$ : «'abandonne le journal intime (qui n'était pas si intime, j'avais les mots des autres, pas les miens). Je ne m'intéresse pas.» ${ }^{5}$ Ce moi raconté dans Mes Algéries en France prend les allures d'un vaste paysage intellectuel, axiologique, ouvrant vers des destinées plurielles, déjouant même la téléologie du «devenir écrivain» qui imprime son sceau sur toute entreprise autobiographique. Or, écrire une autobiographie n'est pas seulement exposer le cours de sa vie passée, c'est aussi poser une sorte de prise du pouvoir, un acte de conquête de la figure même de l'écrivain - son autorité, au double sens de statut d'auteur et d'aptitude à en imposer telle qu'une langue et une culture données l'ont élaborée. «Dire "Je”, l'écrire, ça

\footnotetext{
${ }^{4}$ SEBBAR. L'Arabe comme un chant secret, p. 59.

${ }^{5}$ SEBBAR. L'Arabe comme un chant secret, p. 55.
} 
s'apprend», ${ }^{6}$ écrit-elle encore dans L'Arabe comme un chant secret. Une prise du pouvoir symbolique que Leïla Sebbar réalise tout en tempérant ses effets dogmatiques ou violents, grâce aux vertus de l'iconotexte, justement. Il permet une circulation des images d'un chapitre à l'autre, tantôt personnelles, tantôt culturelles, tantôt documentaires, tantôt esthétiques, qui ne valent que différentiellement, dans un brassage donné à travailler, à recouper par le lecteur. A lui de recoudre, à son tour, entre elles les pièces qui font signe, qui murmurent une langue que tant de conflits ont brouillée, mais qui pourtant est là, familière, intimement lovée.

Une langue à imaginer sans doute mais pas immatérielle pourtant. Pour donner y compris à celles et ceux à qui cette histoire est étrangère - à penser et à voir une discorde entre des peuples et une discordance entre deux langues, paternelle et maternelle, prises au fil d'une histoire dont le cours n'est pas terminée, Leïla Sebbar relie et rallie mots et images. Doit être lié tout ce qui a été délié: «Je n'oublie pas l'image, tout ce qui fait signe, les traces mémorielles qui jouent sur des correspondances insolites, imprévues, troublées», ${ }^{7}$ écrit-elle encore dans L'arabe comme un chant secret. «Je suis dans les livres de la langue maternelle, dans le symbole institutionnel de la langue écrite pour toujours dans le labyrinthe de la bibliothèque.» ${ }^{8}$ L'iconotexte est la forme qui permet de relater une histoire de haine et d'amour qui ne sait plus comment dépasser l'amnésie des uns ni l'hypermnésie des autres, qui permet d'écrire d'un point de vue non autoritaire, de marquer une réserve, juste assez pour qu'il s'agisse d'une manière d'être dans la langue maternelle, le français, langue d'une littérature prestigieuse, sans, cependant, quitter ni trahir la discrète musique de la langue du père dont elle ne saurait user et qu'elle peut seulement faire entendre comme une basse continue.

Cette manière non autoritaire d'incarner l'écrivain, cette forme «altérocentrique» de l'autobiographie, c'est ce dispositif iconotextuel qui permet aux images de jouer pleinement leur propre jeu comme signes, esquissant une parole à nouer aux blancs des discours. Les images ne s'inscrivent pas en illustration des mots, ni en complément de paroles moins parlantes qu'assourdissantes. Muettes, elles figurent justement la basse continue de la langue du père, elles en marquent la trace et en réservent la place, un peu à la façon dont chez Stendhal la langue de la mère, l'italien, jour le rôle de cette tierce langue. Elles ne sont donc nullement extra-littéraires. Les images de cet objetlivre sont au contraire le procédé littéraire par lequel Leïla Sebbar fait entrer en dialogisme l'alter avec l'ego et s'ouvre à celles et ceux à qui les discours officiels n'ont pas accordé la parole, ou qui n'en ont pas trouvé une qui sonnât juste. Au lieu de prétendre en imposer avec un "grand récit» fallacieux, Leïla Sebbar s'impose là où se reconnaît un véritable écrivain: dans l'intimité d'un langage original qui n'est qu'à elle pour mieux résonner en chacun.

\section{A}

\footnotetext{
${ }^{6}$ SEBBAR. L'A rabe comme un chant secret, p. 54.

${ }^{7}$ SEBBAR. L'Arabe comme un chant secret, p. 64.

${ }^{8}$ SEBBAR. LA rabe comme un chant secret, p. 27.
} 


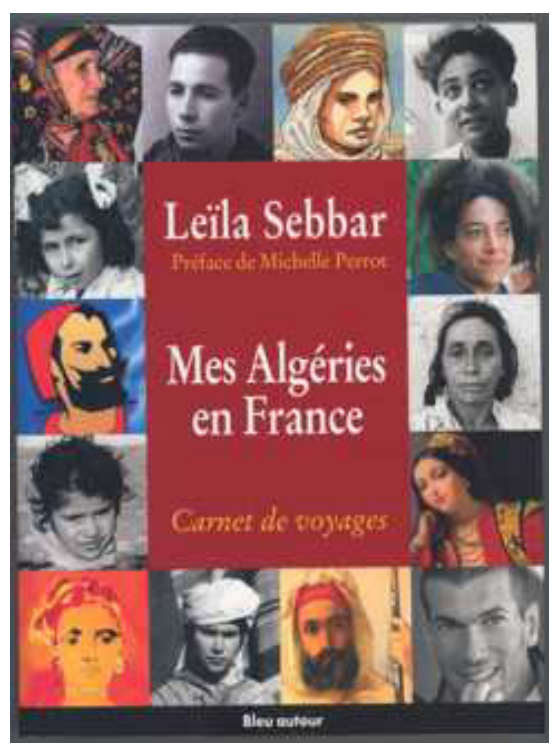

FIGURE 1 - Mes Algéries en France

\begin{abstract}
A B STRACT
In Mes Algéries en France, Leïla Sebbar uses the form of the iconotext for its hybridity, a mirror of her own «variegated» genealogy. Like a glove turned inside out, she uses this 'bastard' genre to underline the dual need to stitch up the wounds opened by history between the families on both sides of the Mediterranean, and to reconceptualise the telling of a particular life as linked or «sewn» into the fabric of all other lives. History and literary autobiography converge in this unique book to invent a new form of autobiography, which I call «alteregotistical», and perhaps peculiar to the figure of the female writer, but also I believe linked to a particular use of photography, whose potential for preserving the past concerns not only the person or the family. Here we see Leïla Sebbar using photography in order to contain a veritable «lieu de mémoire», in the sense that the historian Pierre Nora uses the term.
\end{abstract}

\title{
KEYWORDS
}

Autobiography, iconotext, post-colonial, Leïla Sebbar, photoliterature

\section{RÉ FÉRENCES}

SEBBAR, Leïla. L'Arabe comme un chant secret. Saint-Pourçain-sur-Sioule: Éditions Bleu Autour, 2007.

SEBBAR, Leïla. Mes Algéries en France. Saint-Pourçain-sur-Sioule: Éditions Bleu Autour, 2004. SEBBAR, Leïla. Une enfance outremer. Textes réunis par Leïla Sabbar. Paris: Seuil, 2001. (Points Virgule).

Recebido em 25 de julho de 2014 Aprovado em 1 de outubro de 2014 